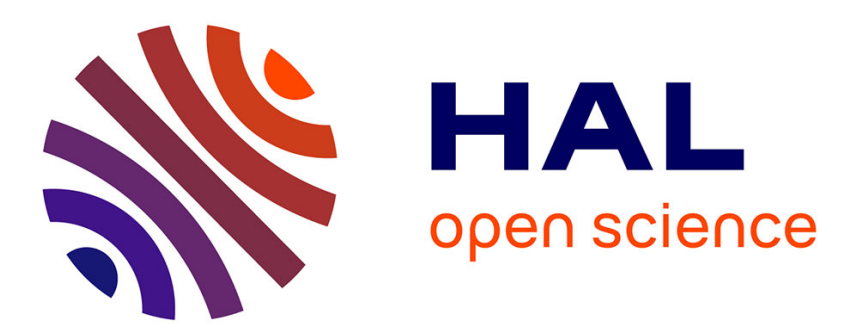

\title{
Analysis of laser induced acoustic pulse probing of charge distributions in dielectrics
}

C. Alquié, J. Lewiner, G. Dreyfus

\section{To cite this version:}

C. Alquié, J. Lewiner, G. Dreyfus. Analysis of laser induced acoustic pulse probing of charge distributions in dielectrics. Journal de Physique Lettres, 1983, 44 (4), pp.171-178. 10.1051/jphyslet:01983004404017100 . jpa-00232176

\section{HAL Id: jpa-00232176 https://hal.science/jpa-00232176}

Submitted on 1 Jan 1983

HAL is a multi-disciplinary open access archive for the deposit and dissemination of scientific research documents, whether they are published or not. The documents may come from teaching and research institutions in France or abroad, or from public or private research centers.
L'archive ouverte pluridisciplinaire HAL, est destinée au dépôt et à la diffusion de documents scientifiques de niveau recherche, publiés ou non, émanant des établissements d'enseignement et de recherche français ou étrangers, des laboratoires publics ou privés. 
Classification

Physics Abstracts

$06.30 \mathrm{~L}-72.20-77.50-42.60-77.30$

\title{
Analysis of laser induced acoustic pulse probing of charge distributions in dielectrics
}

\author{
C. Alquié, J. Lewiner and G. Dreyfus \\ Laboratoire d'Electricité Générale, \\ Ecole Supérieure de Physique et de Chimie Industrielles de la Ville de Paris, \\ 10, rue Vauquelin, 75005 Paris, France
}

(Reçu le 17 novembre 1982, accepté le 23 décembre 1982)

\begin{abstract}
Résumé. - Trois aspects de la méthode de détermination des distributions de champs ou de charges électriques dans les diélectriques, utilisant une onde de pression créée par un laser pulsé sont analysés. 1) Une méthode d'analyse numérique peut être utilisée qui conduit à une résolution spatiale ne dépendant que du temps de montée de l'onde de pression et non de sa durée. Des mesures sont présentées, qui illustrent cette analyse. 2) Les mécanismes de création de l'onde de pression sont étudiés; un dispositif est décrit qui permet de produire des centaines d'impulsions de pression sans variation notable de la forme de ces impulsions, avec un bon rendement de conversion d'énergie. 3) L'influence des propriétés élastiques du matériau sur l'atténuation et la dispersion de l'onde est considérée. Des mesures effectuées sur des échantillons de téflon montrent que ces deux effets ne peuvent pas être négligés si le temps de montée de la pression est beaucoup plus court que le temps de transit de l'onde dans l'échantillon.
\end{abstract}

\begin{abstract}
Some features of the laser induced acoustic pulse method for the determination of electric field or charge distributions in dielectrics are analysed. Three aspects of this method are described. 1) The electric field can be derived by a numerical method whose resolution depends essentially on the rise time of the pressure wave. Measurements are described which support this analysis. 2) The mechanisms of generation of the pressure wave are studied; a method is described which allows the production of hundreds of pulses without noticeable alteration, with a good energy conversion efficiency. 3) The influence of the elastic properties of the material on the attenuation and dispersion of the wave is considered. Measurements performed on Polyfluoroethylenepropylene (FEP) samples show that one has to take these parameters into account if the rise time of the pressure is much shorter than the transit time.
\end{abstract}

1. Introduction. - During the last few years, new approaches to the study of charge storage and transport phenomena in dielectrics have been proposed with a particular interest in the determination of the spatial distribution of electric charges or fields inside the material.

Three methods [1-3] are being currently developed. They all use the diffusion or propagation of an inhomogeneous perturbation of the charges.

First Collins [1] proposed to use the inhomogeneous strain field associated with the diffusion of heat pulses in the sample; this method, known as the «thermal pulse method » can give the 
localization of the charge centroid and a few coefficients of the distribution [4]. The « electronbeam " method proposed by Sessler et al. [3] permits a high resolution determination of the distribution but is destructive and is not applicable to all materials since it implies assumptions as to the conduction mechanisms involved.

In 1977, we proposed [2] to use the propagation of a step function pressure wave to produce an inhomogeneous deformation of a sample and showed that the induced voltage or charge variation are directly related to the spatial distribution of the potential inside the sample. Several attempts were made to generate such a pressure discontinuity using shock tubes [5], spark-gap [6] and quartz crystal [7] techniques. The first two methods were limited by the difficulty of creating reproducible short-rise time perturbation and the spatial resolution obtained was typically $100 \mu \mathrm{m}$. The electrical excitation of a quartz crystal proposed by Eisenmenger [7] is more successful, it leads to a good resolution (a few microns).

Two important steps were made very recently, stimulating interest in the acoustic pulse method. First it was shown $[6,8]$ that an ideal step-function pressure profile is not compulsory since if the shape of the pressure wave is known, the electric field distribution can be derived from the observed signal through a mathematical derivation which leads to a unique solution. Second, the impact of a short-duration laser pulse incident on an absorbing target produces short-rise time pressure pulses which can be transmitted in various ways to the sample under investigation [8-11].

In the case of references [9] and [10], since the duration of the laser pulse is respectively $25 \mathrm{~ns}$ and $15 \mathrm{~ns}$, the spatial resolution is not better than $50 \mu \mathrm{m}$ and the analysis is restricted to a few $\mathrm{mm}$ thick samples. By using much shorter laser pulses (1.5 ns in Ref. [8], 75 ps to $1 \mathrm{~ns}$ in Ref. [11]), a higher resolution is obtained and the technique is applicable to thin film samples.

In this paper, we discuss several àspects of this laser induced pressure pulse method for the determination of field and charge distribution in dielectrics.

First we analyse the various methods which have been proposed to derive electric field or charge distributions from the observed signals, depending on the spatial extent of the pressure wave and the thiekness of the sample and show that if the shape of the pressure pulse is accurately measured, the spatial resolution of the method is related to the rise time of this pulse but does not depend critically on its duration. Then we describe varioús experimental configurations which can be used to produce short-rise time pressure waves using a pulsed laser and characterize the corresponding pressure wave. Finally, we show that in many situations, the attenuation and dispersion of the pressure wave must be taken into account. This is illustrated by data obtained with FEP samples.

2. Analysis of the data. - It has been shown $[2,8]$ that the electric field distribution inside a dielectric sample can be derived from the time dependence of the open-circuit voltage $V(t)$ which appears during the propagation of a pressure wave inside this sample. If the pressure profile is described by $p(z, t)$ and if the dependence of the dielectric constant with pressure is neglected, $V(t)$ is given by :

$$
V(t)=\chi \int_{\mathbf{z}_{\mathbf{f}}}^{d_{0}} E(z, 0) p(z, t) \mathrm{d} z,
$$

where $\chi$ is the compressibility of the sample, $d_{0}$ its thickness, $z_{\mathrm{f}}$ the position of the wave front and $E(z, 0)$ the electric field distribution before penetration of the wave.

A very similar expression was obtained by Migliori [6] taking into account a pressure dependent dielectric constant. This expression was written down explicitly in reference [10] in the case of a dielectric obeying a Clausius Mossotti relation.

The electric field distribution can be numerically derived from the measurements of $V(t)$ and of the pressure profile $p(z, t)$. If the attenuation in the sample can be neglected, it is only necessary to measure the time dependence of the pressure wave before its penetration in the sample. 
This was done in references [8] and [10]. The electric field was derived, in reference [8], by a numerical analysis of the pressure, and in reference [10] by an analytical representation of this pressure. In this later case the pressure was supposed to have a negligible rise time followed by an exponential decay $\mathrm{e}^{-t / t_{0}}$, where $t_{0}=0.388 \mu \mathrm{s}$.

We will now show how, using equation (1) and a limited number of points to describe the pressure rise either numerically or analytically, it is possible to improve the spatial resolution.

As was done in reference [8], we transform the measured pressure $p\left(d_{0}, t\right)$ and the measured signal $V(t)$ into a set of $2 N$ dimensionless values $p_{\mathrm{r}}^{n}, V_{n}$ with $1 \leqslant n \leqslant N$. These values are filtered by a piecewise smoothing algorithm which we have developed for this purpose. Finally, the inversion process is carried out with an iterative improvement, which yields the required values of the electric field. Since the initial values of the pressure and of the measured voltage are critical, the influence of the step $T / N$ on the resolution of the process was investigated systematically. The best results were obtained with a time increment of the order of $0.2 \tau_{\mathrm{r}}$. It can be noticed that by improving either the deconvolution method or the precision of the measurement of $p$ and $V$ at short times the resolution can clearly be improved. For instance, in the case of a pressure profile such as described by curve 4 of figure 1, the optimum time increment was found to be of the order of $1.3 \mathrm{~ns}$, which corresponds to a spatial resolution of $2 \mu \mathrm{m}$ in $25 \mu \mathrm{m}$ thick FEP samples. From this analysis, it is clear that if equation (1) is used in order to derive the electric field distribution in the sample, the spatial resolution is limited by the rise time of the pressure wave and the response time and dynamic range of the electronic circuits. It does not depend critically on the duration of the pressure wave. The charge distribution can be obtained by differentiating the electric field distribution with respect to the space coordinates.

3. Analysis of the generation of laser induced pressure pulses. - The generation of high amplitude pressure waves by the impact of a laser pulse on an absorbing target originates from a rapid heating of the surface of this target. The mechanisms involved in this generation depend on the energy of the pulse, on the absorbing properties of the target and on the mechanical conditions imposed to the absorbing surface, and will be discussed in the following.

The absorbing surface must be made of a material having a high absorption coefficient at the wavelength emitted by the laser, a low thermal conductivity and a low heat of vaporization. It can be for instance a metal as used in references [8-9], or carbon, as in references [10-11]. Aluminum is one of the most commonly used metals. Zinc is also well suited for operation with a Nd-YAlG laser emitting $1.06 \mu \mathrm{m}$ radiation.

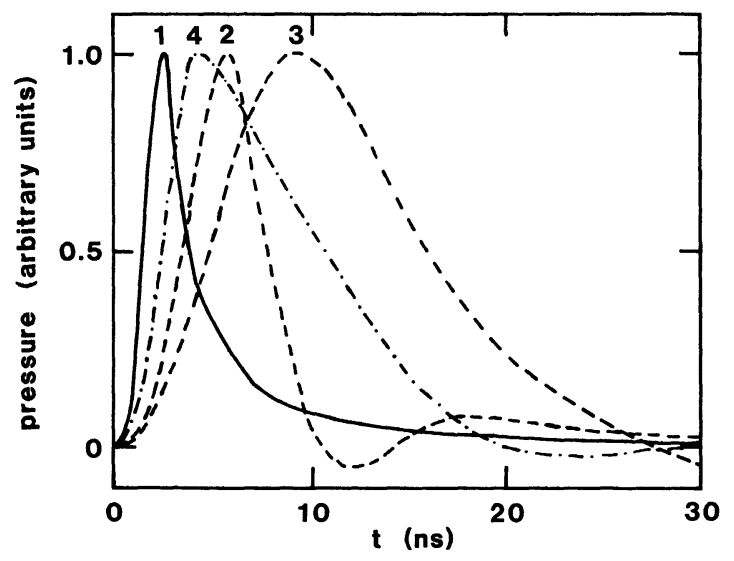

Fig. 1. - Normalized pressure profile $p(t)$ obtained with various targets : 1) evaporated aluminum; 2) and 3) evaporated aluminum covered with an aerolized graphite layer ; 4) $15 \mu \mathrm{m}$ thick aluminum foil. 
The choice of a laser suitable for studying charge distributions in dielectrics is governed by two conditions. First, the homogeneity of the laser beam must be as good as possible to give a good uniformity of the pressure pulse over the whole irradiated area. For this reason, solid-state lasers are preferable to gas lasers. Second, the duration of the laser pulse must be less than a few nanoseconds, in order to permit a resolution of a few microns since if the sound velocity in the sample is $2000 \mathrm{~m} / \mathrm{s}, 2 \mathrm{~ns}$ corresponds to a transit time of $4 \mu \mathrm{m}$. The development of easily available, short-duration (typically 30 ps to a few ns) Nd-YAlG pulsed lasers makes them convenient for the experiments described in this paper. The best spatial homogeneity can be obtained if the last stage of amplification is made of a Nd-glass rod.

We have used a Nd-YAlG laser from QUANTEL, emitting $7 \mathrm{~mJ}$ to $350 \mathrm{~mJ}$ in $3 \mathrm{~ns}$, at $1.06 \mu \mathrm{m}$, with a beam diameter of $8 \mathrm{~mm}$. The same kind of laser, emitting shorter pulses ( $75 \mathrm{ps}$ to $1 \mathrm{~ns})$ is used by Sessler et al. [11].

The time dependence of the pressure pulse, which can be characterized by its rise time $\tau_{\mathrm{r}}$ and its full width at half magnitude (FWHM), may differ significantly from that of the laser, and is influenced by the thickness of the target and the coupling between the target and the sample. Moreover the duration of the pulse depends strongly on the energy of the laser pulse and on the environment of the absorbing surface.

In the simplest configuration, the laser beam impinges on a bare surface in air. In this case, the width of the pulse is related to the properties of the ionized plasma created near the absorbing surface by evaporation of a small amount of material. A power density of the order of $10^{8} \mathrm{~W} / \mathrm{cm}^{2}$ is generally necessary to produce this evaporation. This type of mechanism has been used by various authors $[8,9,11]$, either with a target bounded to the sample or on targets directly deposited on it. This last method has the advantage of avoiding a coupling layer between the sample and the target and leads to very short duration pressures pulses [11]; however it may induce some new problems as is shown in the following.

We have measured, using a piezoelectric quartz transducer as already mentioned in reference [8], the pressure profile produced by a $3 \mathrm{~ns}$ laser pulse incident on an aluminized quartz covered with an aerolized graphite layer. The normalized pressure obtained for a power density of $0.14 \mathrm{GW} / \mathrm{cm}^{2}$ is shown on figure 1 . For a bare aluminum film (curve 1), the width of the pulse is the same as that of the light pulse. Decreasing the duration of the light pulse decreases correspondingly the width of the pressure pulse, thereby increasing the resolution of the method. Unfortunately the aluminum film is destroyed by a single pulse, so that it cannot be used as a convenient target. When the aluminum film is protected by a graphite layer (curves 2 and 3 ), the irregularities of the thickness of the film, which are difficult to control, produce a broadening of the pulse which depends on the quality of the coating and varies when repetitive shots are applied due to the evaporation of the layer.

To be applicable to the study of the evolution of charge distributions in dielectrics, it is necessary to control the quality and repeatability of the absorbing layer.

For comparison, we show on curve 4 of figure 1 the pressure obtained at the rear surface of a $15 \mu \mathrm{m}$ thick aluminum foil irradiated in air, and bounded to the transducer with Nonacq grease. The thickness of this target is sufficient to allow a large number of shots without noticeable variations of its thickness. Nevertheless its surface must be slightly abraded in order to increase the absorption of the laser beam. This leads to a measured peak pressure amplitude of 80 bars for a power density of $0.14 \mathrm{GW} / \mathrm{cm}^{2}$, instead of typically 500 bars for an aerolized graphite target.

In order to improve the conversion from electromagnetic to mechanical energy, it is possible to cover the target with a transparent overlay, thus constraining the absorbing surface [12]. The local and rapid thermal expansion of this layer creates a large gradient of displacement and consequently generates a stress wave. In this case the energy of the laser pulse can be considerably reduced, and it is not necessary to evaporate a part of the target in order to obtain peak pressures of a few tens of bars. Such an effect is used by Migliori [10], who describes an experimental set-up suitable for studying large dimensions samples. 
For small dimensions samples, we have obtained very reproducible results with the set-up described on figure $2 a$. An absorbing layer of colloidal graphite (Aquadag) is covered with a $3 \mathrm{~mm}$ thick fused quartz plate which is transparent at $1.06 \mu \mathrm{m}$. The thickness of this layer, which provides a good energy transmission to the sample, is controlled using a $10 \mu \mathrm{m}$ thick spacing ring against which the sample or the transducer are pressed. The corresponding pressure profile, obtained with only $5 \mathrm{~mJ}$ pulses, and a power density of $3 \times 10^{6} \mathrm{~W} / \mathrm{cm}^{2}$, is shown on figure $2 b$; the rise time is $2.8 \mathrm{~ns}$ and the FWHM is $5 \mathrm{~ns}$. In these conditions no evaporation occurs so that the shape and the amplitude of the pressure pulse are perfectly reproducible, even after hundreds of shots, making signal averaging techniques possible. Moreover these results remain constant from one mounting to another.

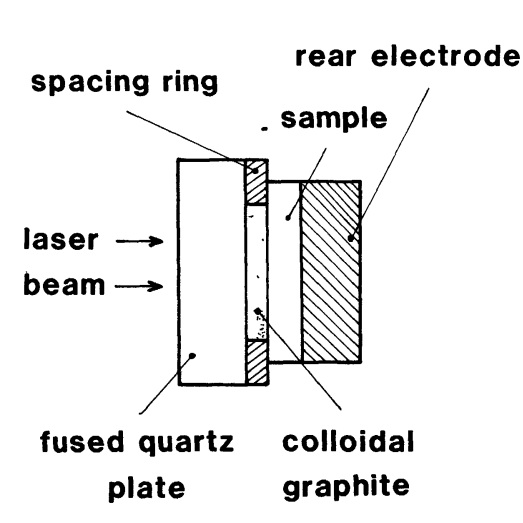

(a)

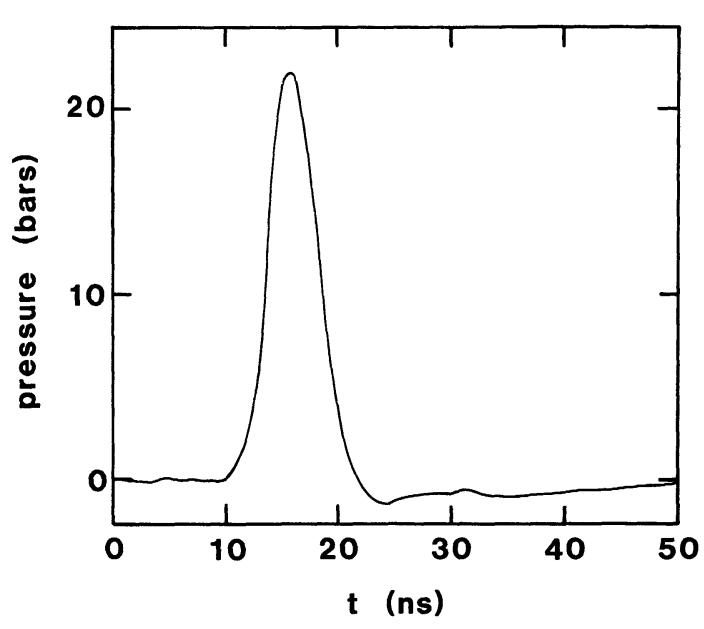

(b)

Fig. 2. - Pressure wave obtained with a confined layer of colloidal graphite. a) Schematic drawing of the target assembly; $b$ ) Time dependence of the corresponding pressure.

4. Influence of the attenuation of the pressure wave. - During the propagation in the dielectric material, the pressure wave profile is altered, depending on the elastic properties of the material. The time dependence of the pressure wave before entering the sample can be expressed in terms of its Fourier components $p\left(d_{0}, \omega\right)$ as :

$$
p\left(d_{0}, t\right)=\frac{1}{2 \pi} \int_{-\infty}^{+\infty} p\left(d_{0}, \omega\right) \exp (i \omega t) \mathrm{d} \omega .
$$

If the attenuation and dispersion of the electric waves in the sample are neglected, all these components propagate at the same velocity $v$, so that in a plane located at $z$, the Fourier component of the pressure distribution, of pulsation $\omega$, becomes :

and

$$
p(z, \omega)=p\left(d_{0}, \omega\right) \exp -\left[i \omega\left(d_{0}-z\right) / v\right]
$$

$$
p(z, t)=\frac{1}{2 \pi} \int_{-\infty}^{+\infty} p(z, \omega) \exp (i \omega t) \mathrm{d} \omega=p\left[d_{0}, t-\left(d_{0}-z\right) / v\right]
$$


In this case, the pressure wave propagates in the sample without any deformation of its profile.

When attenuation and dispersion occur, the phase velocity $v$ becomes frequency dependent and is related to the attenuation $\alpha(\omega)$ by Kramers-Kronig relationship [13]. A good approximation of this relation, which is valid in many cases, is given in reference [13] by :

$$
\alpha(\omega)=\frac{\pi \omega^{2}}{2 v_{0}^{2}} \frac{\mathrm{d} v(\omega)}{\mathrm{d} \omega} \quad v(\omega)-v_{0}=\frac{2 v_{0}^{2}}{\pi} \int_{\omega_{0}}^{\omega} \frac{\alpha\left(\omega^{\prime}\right)}{\omega^{\prime 2}} \mathrm{~d} \omega^{\prime}
$$

where $v_{0}$ is the sound velocity at a reference pulsation $\omega_{0}$. In these conditions, the pressure profile $p(z, t)$ becomes :

$$
p(z, t)=\frac{1}{2 \pi} \int_{-\infty}^{+\infty} p\left(d_{0}, \omega\right) \exp -\left[\alpha(\omega)\left(d_{0}-z\right)\right] \exp i \omega\left[t-\left(d_{0}-z\right) / v(\omega)\right] \mathrm{d} \omega .
$$

Knowing its elastic properties, this expression completely describes the evolution of the pressure distribution inside a material. In order to be used, it requires that ultrasonic attenuation and sound velocity data are available, in the frequency range for which the Fourier components of the pressure profile are large. If this is not the case, it is however possible to evaluate simply the influence of attenuation and dispersion on the pressure, by measuring the pressure transmitted through samples of increasing thicknesses.

We have performed such an analysis on F.E.P. samples. The pressure wave was produced using a $\mathrm{CO}_{2}$ laser, as already described in reference [8], and was transmitted to the samples by a bonding layer (conductive adhesive $E$ solder 3021). The influence of this layer has been taken into account by comparing the pressure obtained directly at the rear surface of the target (curve $a$ of Fig. 3) to that transmitted through $12.5 \mu \mathrm{m}$ thick samples (curve $b$ ).

In this case we have assumed that the attenuation and broadening of the pressure wave are caused by the bonding; the measurements show that the amplitude of the pressure wave decreases typically in a $4: 1$ ratio and the maximum of the pressure occurs at about $15 \mathrm{~ns}$ instead of $9 \mathrm{~ns}$. Curves $c$ and $d$ of figure 3 are the pressure waves obtained after propagation through $125 \mu \mathrm{m}$ and $250 \mu \mathrm{m}$ thick samples. The dispersion and attenuation of the high frequency components of the

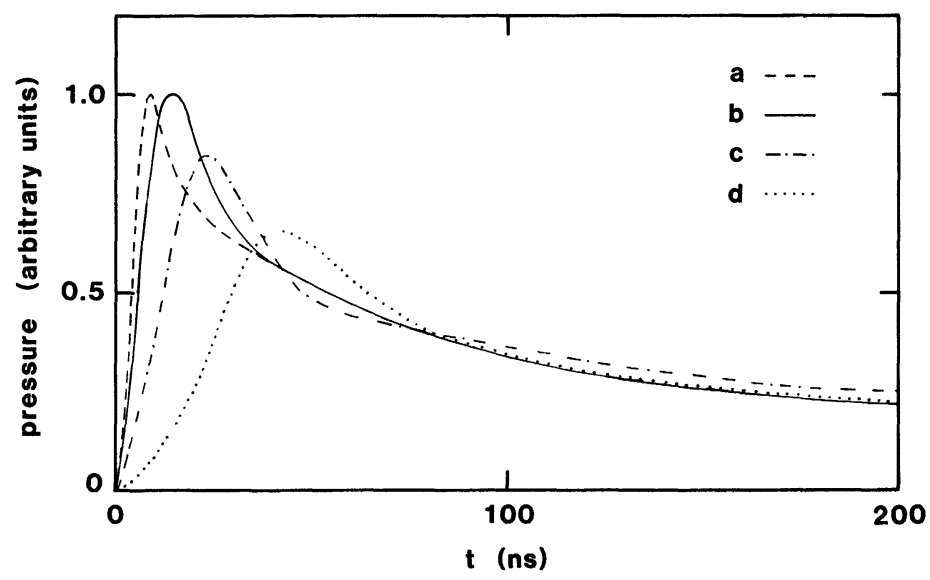

Fig. 3. - Time dependence of the pressure transmitted through F.E.P. samples of different thicknesses. a) Directly at the rear surface of the target. In order to be comparable to the other curves, the amplitude was divided by 4 to take into account the influence of the bonding layer; $b$ ) after $12.5 \mu \mathrm{m} ; c$ ) after $125 \mu \mathrm{m}$; d) after $250 \mu \mathrm{m}$. 
pressure wave propagating in teflon produce an increase of the rise time and a broadening of the pressure pulse which cannot be neglected for $125 \mu \mathrm{m}$ thick samples.

We have used these results to analyse the distribution of the electric field which was created in a $250 \mu \mathrm{m}$ thick F.E.P. film. The sample was metallized on one side, charged by a negative corona process, heated for a few hours to broaden and stabilize the distribution. It was then metallized on the other side and bonded on both sides to $1.5 \mathrm{~mm}$ thick aluminum plates so that the whole assembly was completely symmetrical and the pressure wave could be created either near the charged surface or near the opposite one. The open circuit voltage was measured for a propagation of the pressure wave in both directions and the electric field distribution was calculated from equation (1). In a first derivation the attenuation was neglected and the pressure profile $p(z, t)$ was derived from curve $b$ of figure 3 , which was obtained in a similar experimental arrangement. The corresponding electric fields are shown on figure $4 a$. In two regions extending over $70 \mu \mathrm{m}$ near the surfaces, a discrepancy appears between the distributions obtained for the two directions of propagation of the wave.

This can be explained by the fact that the attenuation of the pressure wave at the end of its transit through the sample is not negligible. In order to check this assumption, we have constructed the matrix $p\left(z_{i}, t_{j}\right)$ using curves $b, c$ and $d$ of figure 3 . When the wave front propagates from 0 to $125 \mu \mathrm{m}$, the pressure profile is linearly interpolated from curve $b$ to curve $c$, while in the second part of the sample, it varies linearly from curve $c$ to curve $d$. In these conditions, the electric field distributions derived from equation (1) are shown on figure $4 b$. A good agreement is obtained, for the two directions of propagation of the wave, even near the faces of the sample.

This clearly shows that in F.E.P. samples, the attenuation of the high frequency components of the pressure wave must be taken into account for thicknesses larger than $125 \mu \mathrm{m}$. If very short pressure pulses are used, higher frequency components are involved and are even more quickly attenuated, so that attenuation and dispersion will have to be considered for thinner samples.

In reference [11], Sessler et al. proposed a method for measuring directly the charge distribution, using a very short duration pressure pulse. Assuming that the spatial extent of the pulse is very short compared to any Fourier component of the charge distribution, they obtain this distribution from the short-circuit current flowing in the external circuit during the propagation of the pressure wave in the sample. This very simplified model is only valid if the duration of the pressure pulse $\tau$

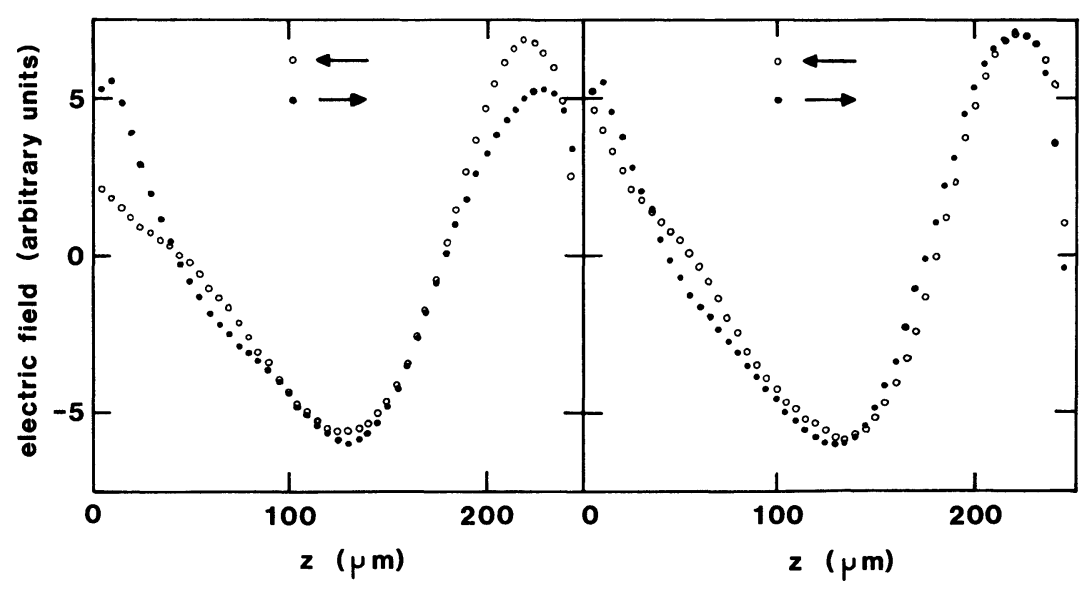

(a)

(b)

Fig. 4. - Electric field distributions obtained from the measurement of the open-circuit voltage, for two opposite directions of propagation of the pressure wave. a) Neglecting the attenuation and dispersion in the sample. $b$ ) Taking these effects into account. 
before entering the sample is such that $v \tau$ corresponds to a sufficient resolution. Moreover, from what we have just shown, it is restricted to rather thin samples, in which pulse attenuation and broadening during propagation in the dielectric can be neglected.

By measuring the pulse broadening of pressure waves such as shown on curve 2 of figure 1, after a transit through $25 \mu \mathrm{m}$ and $125 \mu \mathrm{m}$ F.E.P. samples, we have evaluated the maximum thickness of F.E.P. films associated with the above limitation to be in the range of $50 \mu \mathrm{m}$.

5. Conclusion. - In this paper, we have analysed some important features of the laser-induced pressure pulse method for the investigation of electric field or charge distributions in dielectrics. We have shown that, when the electric field is derived from the signal measured during the propagation of the pressure wave and from the pressure profile, using a numerical method, it is possible to obtain by an appropriate choice of the sampling interval, a resolution better than $v \tau_{r}$, where $\tau_{\mathrm{r}}$ is the rise time of the pressure wave and $v$ the sound velocity.

We have then analysed the generation of pressure waves using pulsed lasers and have shown that the energy of the laser can be considerably reduced if the absorbing surface is constrained. Because in this case the absorbing material is not evaporated by the impact of the laser, the reproducibility of the pressure wave is much better than in the case of a free surface.

Finally we have studied the influence of the elastic properties of the material on the pressure distribution in rather thick samples. In order to obtain the field distribution with a good resolution, it is necessary to take into account the attenuation and dispersion of the high frequency components of the pressure wave.

We have shown that in F.E.P. samples, for pressure waves having a rise time of $8 \mathrm{~ns}$, these effects must be taken into account for thicknesses of the order of or larger than $125 \mu \mathrm{m}$. More generally, it appears that in F.E.P. the broadening of the pressure pulse is important if the transit time through the sample is one order of magnitude larger than the rise time of the pulse.

Should that be a limitation, it would be possible to reduce the attenuation of the pressure wave by operating at low temperatures.

\section{References}

[1] Collins, R. E., J. Appl. Phys. 47 (1976) 4804.

[2] Laurenceau, P., Dreyfus, G. and Lewiner, J., Phys. Rev. Lett. 38 (1977) 46.

[3] Sessler, G. M., West, J. E., Berkeley, D. A. and Morgenstern, G., Phys. Rev. Lett. 38 (1977) 368.

[4] Von Seggern, H., Appl. Phys. Lett. 33 (1978) 134 ; MopsiK, F. I. and De RegGi, A. S., J. Appl. Phys. 53 (1982) 4333.

[5] Laurenceau, P., Ball, J., Dreyfus, G. and Lewiner, J., C. R. Hebd. Séan. Acad. Sci. ser. B 283 (1976) 135.

[6] Migliori, A. and Thompson, J. D., J. Appl. Phys. 51 (1980) 479.

[7] EISENMENGer, W. and HAARDT, M., Solid State Commun. 41 (1982) 917.

[8] Alquie, C., Dreyfus, G. and Lewiner, J., Phys. Rev. Lett. 47 (1981) 1483.

[9] Rozno, A. G. and Gromov, V. V., Pis'ma Zh. Tekh. Fiz. 5 (1979) 648 (Sov. Tech. Phys. Lett. 5 (1979) 266).

[10] Miglori, A. and Hofler, T., Rev. Sci. Instrum. 53 (1982) 662.

[11] Sessler, G. M., West, J. E. and Gerhard, R., Phys. Rev. Lett. 48 (1982) 563.

[12] Von Gutfeld, R. J. and MelCher, R. L., Appl. Phys. Lett. 30(1977) 257.

[13] O'Donnfll. M.. JAYnes. E. T. and Miller, J. G., J. Acoust. Soc. Am. 69 (1981) 696. 